This file contains Supporting Methods, supporting Tables 1, 3, 4, and Supporting Figures 2, 3. Supporting Table 2 and Figure 1 are separate files due to their size.

\title{
Supporting Methods
}

pTLJ02 construction. Plasmid pTLJ02 was derived from Gateway ${ }^{\mathrm{TM}}$ destination vectors 15 and 17 (Invitrogen, Carlsbad, CA). It contains GST followed by an enterokinase cleavage site (EK) and a 6xHis tag immediately upstream of the attB1 site. The following steps were performed to construct plasmid pTLJ02. First, PCR was performed to generate a GST-EK fragment flanked by NdeI restriction sites. Plasmid pDES15 (Invitrogen, Carlsbad, CA), which contains GST, was used as template. The 5' NdeI site was already present in pDES15 while the 3' NdeI site was introduced in the primer (see underlined sequence below). Primer sequences are as follows: primer336, 5'GGG AAT TCC ATA TGT CCC CTA TAC TAG GTT ATT GGA AAA TTA AGG GC CTT3' and primer343, 5'GGG AAT TCC ATA TGA TCC TTA TCG TCA TCG TCC GAC CAT GGA CGC GGA AC3'. Secondly, the generated PCR product was subcloned into pGEM-T Easy vector (Promega, Madison, WI) as per manufacturer's instructions, generating plasmid pM3. Plasmid pM3 was digested with NdeI and the resulting 700bp NdeI-GSTEK-NdeI fragment gel-purified (Qiagen, Valencia, CA) and ligated into plasmid pDES17 (Invitrogen, Carlsbad, CA) at the NdeI site immediately upstream of the His tag. Plasmid DNA was verified for the insert by restriction enzyme digestion and sequencing. We sequenced four resulting clones (pM6-pM9) and found that all contained a deletion of "G" in the junction between GST and EK, thereby creating a new SalI (GTCGAC) site that we confirmed by SalI restriction digest. The expected region should be GTCGGAC. This problem will result in a frame shift affecting downstream translation, therefore it was necessary to correct the mistake. To accomplish this we used plasmid pM6 as a template for PCR amplification of a $814 \mathrm{bp}$ fragment containing the 3' end of GST (including the NcoI site), the EK site, the His tag, the 5' attR sites, CapR, and the flanking $N c o$ I site with the following primers: primer390, 5' GGA TCA ACT GCC ATG GTC GGA CGA TGA CGA TAA GGA TCA T3' and primer392, 5' CTT GTC GCC TTG CGT ATA ATA TTT GCC CAT GGT GAA AAC G3'. The new PCR product does not contain the SalI (GTCGAC) site but has the expected GTCGGAC sequence instead. The PCR product was then digested with NcoI. Meanwhile, pDES15, which contains the T7 promoter and GST Tag, was digested using NcoI generating two bands of 6.2-kb and $750 \mathrm{bp}$. The $6.2 \mathrm{~kb}$ band was gel extracted, treated with CIAP (Promega, Madison, WI), and then ligated with the 814 bp EK-His fragment. A resulting clone containing the correct insert was verified by sequencing and named, pTLJ02. pTLJ02 is a functional destination vector for cloning via the Gateway ${ }^{\mathrm{TM}}$ system (data not shown). 


\section{Supporting Table 1. E. coli KC8, BUN10, and MG1655 recombinational cloning efficiencies.}

\begin{tabular}{|c|c|c|c|c|c|c|}
\hline & \multicolumn{3}{|c|}{$19,20 \mathrm{bp} \mathrm{tags}^{\mathrm{a}}$} & \multicolumn{3}{|c|}{36,41 bp tags } \\
\hline & $\begin{array}{c}f o l E \\
(573 \mathrm{bp})^{\mathrm{b}}\end{array}$ & $\begin{array}{c}f t s Z \\
\text { (1113bp) }\end{array}$ & $\begin{array}{c}f l i A \\
(717 \mathrm{bp})\end{array}$ & folE & $f t s Z$ & fliA \\
\hline KC8 & $75 \%(4)^{c}$ & $67 \%(3)$ & $75 \%(4)$ & $100 \%(11)$ & $91 \%(11)$ & $92 \%(12)$ \\
\hline BUN10 & $0 \%(1)$ & $100 \%(1)$ & $67 \%(3)$ & $67 \%(9)$ & nd & $100 \%(8)$ \\
\hline
\end{tabular}

\begin{tabular}{|c|c|c|c|c|c|}
\hline \multirow{2}{*}{} & \multicolumn{5}{|c|}{$36,41 \mathrm{bp}$ tags } \\
\cline { 2 - 6 } & $\begin{array}{c}\text { cheA } \\
(2310 \mathrm{bp})\end{array}$ & $\begin{array}{c}u v r B \\
(1974 \mathrm{bp})\end{array}$ & $\begin{array}{c}\text { Cj0013 } \\
(1677 \mathrm{bp})\end{array}$ & $\begin{array}{c}\text { Cj1190c } \\
(1380 \mathrm{bp})\end{array}$ & $\begin{array}{c}\text { fliA } \\
(717 \mathrm{bp})\end{array}$ \\
\hline KC8 & $87 \%(15)$ & $86 \%(7)$ & $100 \%(5)$ & $100 \%(4)$ & $100 \%(5)$ \\
\hline MG1655 & $100 \%(5)$ & $100 \%(1)$ & $0 \%(1)$ & $100 \%(4)$ & $100 \%(3)$ \\
\hline
\end{tabular}

${ }^{a}$ Recombinational cloning was performed using pIMBD3 (50 ng) linearized using EcoR1 and BamH1, and PCR-amplified ORFs (50ng) tagged either with 19 (5') and 20 (3') bp tags identical to the pIMBD3 cloning site, or 36 (5') and 41 (3') bp tags identical to the pIMBD3 cloning site.

${ }^{\mathrm{b}}$ Predicted ORF length.

${ }^{\mathrm{c}}$ The percentage is the cloning efficiency, the fraction of transformants containing vector plus insert, as determined by PCR. The number in parentheses is the number of transformants checked.

Supporting Table 2 is in a separate (Excel) file. 


\section{Supporting Table 3. Summary of sequence validation.}

\begin{tabular}{|l|c|c|c|}
\cline { 2 - 4 } \multicolumn{1}{c|}{} & \multicolumn{3}{c|}{ Clones sequenced $^{\mathbf{a}}$} \\
\cline { 2 - 4 } \multicolumn{1}{c|}{} & Total & Without error & With error $^{\mathrm{b}}$ \\
\hline Round 1 & 1126 & $1051(93.3 \%)$ & $75(6.7 \%)$ \\
\hline Round 2 & 187 & $167(89.3 \%)$ & $20(10.7 \%)$ \\
\hline Total & 1313 & $1218(92.8 \%)$ & $95(7.2 \%)$ \\
\hline
\end{tabular}

Results of sequence analysis of the ORF-containing clones generated in the first round of cloning (Round 1) using PCR products containing $21 \mathrm{bp}$ recombination tags 5RT1 and 3RT1, and the second round of cloning (Round 2), using re-amplified PCR products containing 5RT1, 3RT1, and 23 bases of additional vector sequence (see text). ${ }^{\text {a}}$ The regions sequenced include $16 \mathrm{bp}$ upstream of the 5RT1, the 5RT1, the sequence corresponding to the genespecific region of the primer, and $100 \mathrm{bp}$ of the ORF sequence. ${ }^{\mathrm{b}}$ Errors refer to single base substitutions or small deletions (see Table 1). Most (83) of the 95 error-containing clones had only one error. 
Supporting Table 4. Summary of $C$. jejuni clones examined for expression.

Listed are the identities (some putative) and the predicted sizes of the cloned ORFs that were expressed and analyzed in Figure 2.

\begin{tabular}{|c|c|c|c|c|}
\hline Position & ORF_cloned & bclone_ID & expect_MW_withGST & Comment \\
\hline A01 & wlaE & bclone1087 & 70826.96 & \\
\hline A02 & Cj0930 & bclone1090 & 70173.96 & \\
\hline A03 & Cj1437c & bclone1091 & 71447.96 & \\
\hline A04 & Cj1068 & bclone1093 & 70479.96 & \\
\hline A05 & null & bclone1094 & 73114.96 & \\
\hline A06 & Cj0250c & bclone1095 & 78731.96 & \\
\hline A07 & $\mathrm{mrp}$ & bclone1096 & 69638.96 & \\
\hline A08 & iamB & bclone1097 & 70940.96 & \\
\hline A09 & livk & bclone1098 & 69233.96 & \\
\hline A10 & Cj1607 & bclone1103 & 71208.96 & \\
\hline A11 & Cj0645 & bclone1106 & 72321.96 & \\
\hline A12 & Cj0789 & bclone1107 & 73208.96 & \\
\hline B01 & Cj1412c & bclone1114 & 73717.96 & \\
\hline B02 & thrs & bclone1115 & 99132.96 & \\
\hline B03 & tgt & bclone1116 & 72092.96 & \\
\hline B04 & Cj0846 & bclone1117 & 72367.96 & \\
\hline B05 & cydB & bclone1118 & 71448.96 & \\
\hline B06 & oorA & bclone1119 & 70570.96 & \\
\hline B07 & petC & bclone1120 & 70961.96 & \\
\hline B08 & Cj1294 & bclone1122 & 71968.96 & \\
\hline B09 & Cj1316c & bclone1124 & 73206.96 & \\
\hline B10 & null & bclone1125 & 70956.96 & \\
\hline B11 & bioF & bclone1126 & 73585.96 & \\
\hline B12 & Cj1551c & bclone1127 & 72690.96 & \\
\hline $\mathrm{C} 01$ & Cj1515c & bclone1130 & 72635.96 & \\
\hline $\mathrm{CO2}$ & nhaA1 & bclone1131 & 71923.96 & \\
\hline $\mathrm{CO3}$ & neuC2 & bclone1136 & 73252.96 & deletion $1 \mathrm{bp}, 5^{\prime}$ primer \\
\hline $\mathrm{CO} 4$ & frdC & bclone1139 & 59546.96 & \\
\hline C05 & Cj1215 & bclone1140 & 73345.96 & \\
\hline $\mathrm{C06}$ & thrS & bclone1141 & 99132.96 & \\
\hline $\mathrm{CO7}$ & dapD & bclone1142 & 71879.96 & \\
\hline $\mathrm{C08}$ & moeA & bclone1143 & 72917.96 & \\
\hline C09 & cfa & bclone1145 & 75181.96 & \\
\hline $\mathrm{C} 10$ & Cj1038 & bclone1146 & 72770.96 & \\
\hline $\mathrm{C} 11$ & Cj1040c & bclone1147 & 72593.96 & \\
\hline $\mathrm{C} 12$ & sucC & bclone1148 & 71270.96 & \\
\hline D01 & xseA & bclone1149 & 73606.96 & \\
\hline D02 & Cj1684c & bclone1153 & 73505.96 & \\
\hline D03 & Cj0606 & bclone1157 & 72339.96 & $\mathrm{~mm} \mathrm{~A}$ to $\mathrm{T}$ in $5^{\prime}$ primer, introduces stop codon \\
\hline D04 & Cj1136 & bclone1158 & 75127.96 & \\
\hline D05 & uxaA_prime_14 & bclone1160 & 29518.96 & \\
\hline D06 & $\operatorname{trpB}$ & bclone1166 & 72465.96 & \\
\hline D07 & ald_prime_14 & bclone1167 & 72425.96 & \\
\hline D08 & Cj0198c & bclone1168 & 74223.96 & \\
\hline D09 & $\mathrm{Cj0240c}$ & bclone1169 & 72539.96 & \\
\hline D10 & Cj1413c & bclone1172 & 76457.96 & \\
\hline D11 & folc & bclone1173 & 75565.96 & \\
\hline D12 & ackA & bclone1178 & 73576.96 & \\
\hline E01 & Cj1275c & bclone1183 & 74609.96 & \\
\hline E02 & Cj1245c & bclone1187 & 76357.96 & \\
\hline E03 & pgk & bclone1195 & 73109.96 & \\
\hline E04 & $\mathrm{Cj0172c}$ & bclone1196 & 74462.96 & \\
\hline E05 & Cj0182 & bclone1197 & 76280.96 & \\
\hline E06 & Cj0941c & bclone1198 & 74530.96 & del "GT" in 5' primer \\
\hline E07 & murD & bclone1203 & 74847.96 & \\
\hline E08 & fabF & bclone1208 & 72201.96 & \\
\hline E09 & Cj0463 & bclone1211 & 76374.96 & \\
\hline E10 & pgi & bclone1212 & 75531.96 & \\
\hline E11 & Cj1097 & bclone1213 & 72788.96 & \\
\hline E12 & Cj0015c & bclone1215 & 76827.96 & \\
\hline H01 & Cj0376 & bclone1297 & 62123.96 & \\
\hline $\mathrm{HO2}$ & Cj0935c & bclone1301 & 78308.96 & \\
\hline $\mathrm{HO3}$ & Cj0092 & bclone1303 & 78758.96 & \\
\hline $\mathrm{HO4}$ & Cj0360 & bclone1304 & 78316.96 & \\
\hline H05 & Cj1434c & bclone1305 & 82410.96 & \\
\hline H06 & purF & bclone1308 & 78859.96 & \\
\hline H07 & Cj0934c & bclone1310 & 79914.96 & \\
\hline H08 & ktrB & bclone1315 & 78992.96 & \\
\hline H09 & Cj0203 & bclone1316 & 77775.96 & \\
\hline H10 & Cj0946 & bclone1319 & 81969.96 & \\
\hline $\mathrm{H} 11$ & hemN & bclone1322 & 81926.96 & \\
\hline $\mathrm{H} 12$ & Cj0183 & bclone1323 & 80511.96 & \\
\hline
\end{tabular}


Parrish et al., Supporting Material.

Supporting Figure 1 is in a separate file.

\section{Supporting Figure 2.}

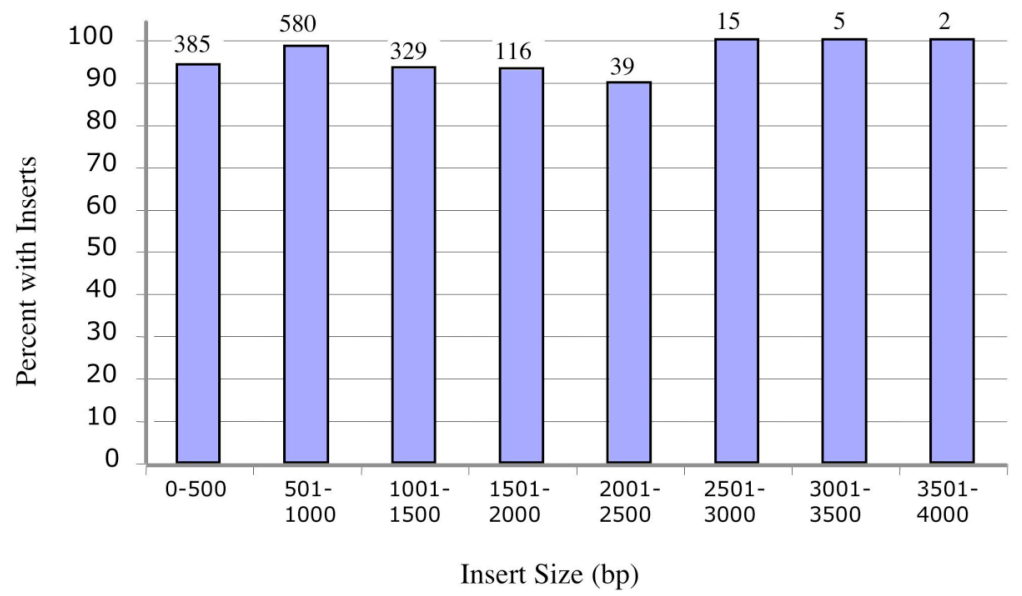

Supporting Figure 2. Comparison of insert size and ORF cloning efficiency. ORF cloning efficiency, defined here as the ability of an ORF to be cloned, appears not to be affected significantly by ORF size. Shown in the histogram are the combined results from Round 1 and Round 2 cloning. The number at the top of each bar corresponds to the number of ORFs within that size range. ORFs that failed to amplify during primary PCR are excluded from this analysis. 
Parrish et al., Supporting Material.

\section{Supporting Figure 3.}

a.

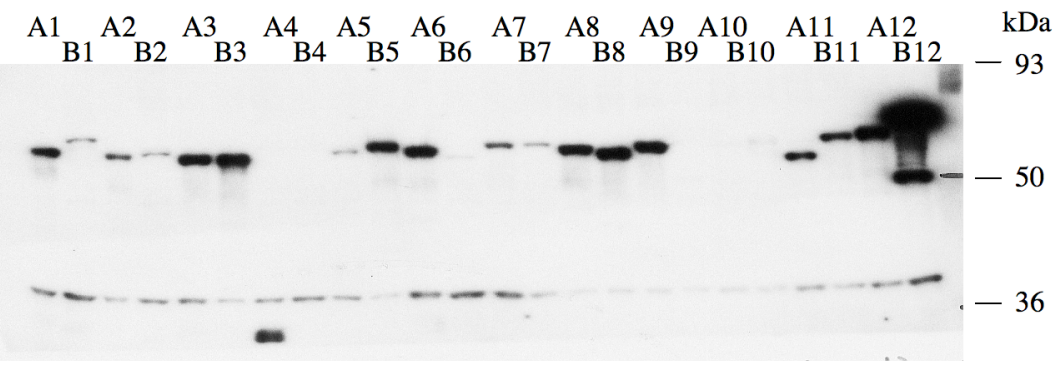

b.

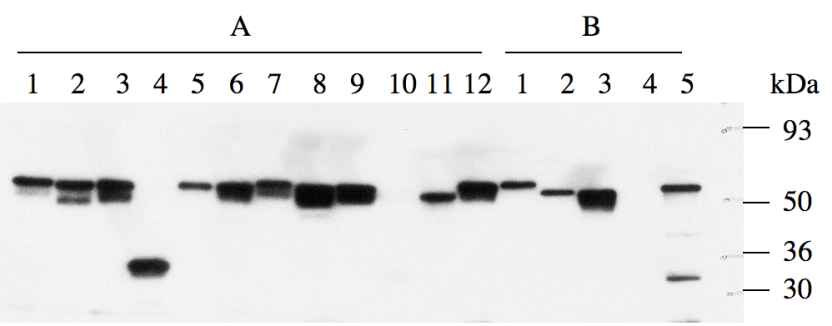

Supporting Figure 3. Western analysis of $C$. jejuni clone expression. Protein expression was detected using anti-His antibodies (panel a) or anti-GST antibodies (panel b). The same extracts were used in both westerns. 